\title{
RECONOCIMIENTO DE ROTURA MIOCÁRDICA EN EMERGENCIA: REPORTE DE CASO
}

\author{
RECOGNITION OF MYOCARDIAL RUPTURE IN EMERGENCY: CASE REPORT \\ Jessica M. Calcino-Cuela ${ }^{1, a}$, Fanny Ramírez-Calderón ${ }^{1, a}$, Rolando Vásquez-Alva 1,2,a,b
}

\begin{abstract}
RESUMEN
Paciente mujer de 86 años, hipertensa que ingresa a emergencia por dolor torácico, disnea, hipotensión y bradicardia con cuadro confusional. El electrocardiograma y el laboratorio es compatible con infarto agudo de miocardio no $Q$, encontrándose en la ecovisión una fracción de eyección ventricular izquierda de $20 \%$ con derrame pericárdico y masa hiperecogénica compatible con coágulo. El diagnóstico planteado fue estado de bajo gasto cardiaco porinfarto de miocardio no $Q$ evolutivo con rotura miocárdica. La paciente requirió intubación endotraqueal y ventilación mecánica, evolucionó con paro cardiaco y fue reanimada sin éxito. Se discute el diagnóstico y manejo oportunos de la rotura cardiaca como una complicación mecánica del infarto agudo miocárdico.
\end{abstract}

Palabras clave: Ecocardiografía; Infarto miocárdico; Rotura miocárdica. (fuente: DeCS BIREME)

\begin{abstract}
A 86-year old female patient with hypertension was admitted to emergency due to chest pain, dyspnea, hypotension and bradycardia with confusional symptoms. The electrocardiogram and the laboratory are compatible with acute non-Q myocardial infarction, with a left ventricular ejection fraction of $20 \%$ in the ultrasound, with pericardial effusion and hyperechoic mass compatible with a clot. The diagnosis was a low cardiac output due to an evolutive non- $\mathrm{Q}$ myocardial infarction with myocardial rupture. The patient required endotracheal intubation and mechanical ventilation, evolved with cardiac arrest and was resuscitated without success. The diagnosis and timely management of cardiac rupture is discussed as a mechanical complication of acute myocardial infarction.
\end{abstract}

Key words: Echocardiography; Myocardial infarction; Myocardial rupture. (source: MeSH NLM)

\section{INTRODUCCIÓN}

La rotura de la pared libre del ventrículo izquierdo es, después del shock cardiogénico, la segunda causa más frecuente de muerte hospitalaria en el infarto agudo de miocardio. Su incidencia ha disminuido en los últimos años por una rápida reperfusión del territorio isquémico y el uso precoz de betabloqueantes e inhibidores de la enzima convertidora de angiotensina, pasando del $6 \%$ en la era preintervencionista hasta menos del $1 \%$ con la aparición de la angioplastia primaria ${ }^{1,2}$. Reportamos el caso de una paciente de 86 años con estado de bajo gasto cardiaco por infarto de miocardio no $Q$ evolutivo con rotura miocárdica.

\section{REPORTE DE CASO}

Paciente mujer de 86 años, raza mestiza con antecedente de hipertensión arterial y bradicardia diagnosticada hace un año. Recibe como tratamiento amlodipino, enalapril, aspirina y simvastatina. Ingresa a emergencia por trastorno de conciencia. Familiar refiere que desde hace algunos días cursa

Hospital Nacional Edgardo Rebagliati Martins, EsSalud, Lima-Perú.

2 Facultad de Medicina Universidad Nacional Mayor de San Marcos, Lima-Perú.

a Médico Emergenciólogo.

${ }^{\mathrm{b}}$ Médico Internista.

Citar como: Jessica M. Calcino-Cuela, Fanny Ramírez-Calderón, Rolando Vásquez-Alva. Reconocimiento de rotura miocárdica en emergencia: Reporte de caso. [Caso Clínico].2019;19(2):00-00. (Abril 2019). DOI 10.25176/RFMH.v19.n2.2076 
con angor de esfuerzo y una hora antes de su ingreso presenta desvanecimiento y disnea.

Al examen clínico presión arterial de 60/40 mm $\mathrm{Hg}$, frecuencia cardiaca en 78 latidos por minuto, frecuencia respiratoria de 30 por minuto, saturación de oxígeno en $80 \%$ y un hemoglucotest de 149 $\mathrm{mg} / \mathrm{dL}$. Al examen físico paciente en mal estado general, somnolienta, con frialdad distal y llenado capilar mayor a 2 segundos, no se evidencia cianosis. Ruidos cardiacos rítmicos de baja intensidad. Soplo sistólico II/VI, ventila espontáneamente con máscara de reservorio, mal patrón respiratorio. Abdomen blando depresible, no se palpa visceromegalia. El examen neurológico sin signos de focalización. Escala Glasglow: 10 puntos (AO 4, RV 2, RM 4).

El electrocardiograma mostró ritmo sinusal, frecuencia de 78 latidos por minuto, con signos de hipertrofia de ventrículo izquierdo, sin elevación del segmento ST, ni presencia de ondas Q (Fig.1). El análisis de gases arterial (AGA) evidenció acidosis metabólica descompensada anión gap normal con lactato en $7.9 \mathrm{mmol} / \mathrm{L} . P h 7.29$, pCO2 21.1 $\mathrm{mmHg}, \mathrm{pO} 225.4 \mathrm{mmHg}, \mathrm{cHCO} 39.9 \mathrm{mmol} / \mathrm{L}$, SO2 99.6\%. En el hemograma leucocitos $9.11 \times 103$ $\mathrm{mm} 3$, hemoglobina $11.3 \mathrm{gr} / \mathrm{dL}$, plaquetas $181000 \mathrm{x}$ $\mathrm{mm} 3$, glucosa $245 \mathrm{mg} / \mathrm{dL}$, urea $24.4 \mathrm{mg} / \mathrm{dL}$, creatinina $0.76 \mathrm{mg} / \mathrm{dL}$, sodio $149.3 \mathrm{mmol} / \mathrm{L}$, potasio $3.46 \mathrm{mmol} / \mathrm{L}$. TGO $378 \mathrm{U} / \mathrm{L}$, TGP $105 \mathrm{U} / \mathrm{L}$, bilirrubina total $0.25 \mathrm{mg} /$ $\mathrm{dL}$, bilirrubina directa $0.17 \mathrm{mg} / \mathrm{dL}$, fosfatasa alcalina $55 \mathrm{U} / \mathrm{L}$, albúmina $2.84 \mathrm{~g} / \mathrm{dL}$, amilasa $206 \mathrm{U} / \mathrm{L}$, lipasa 45 $\mathrm{U} / \mathrm{L}$, tiempo de protrombina $16.44 \mathrm{seg}$. CPK total 31 $\mathrm{U} / \mathrm{L}$, troponina I $7.271 \mathrm{ng} / \mathrm{dL}$. La radiografía de tórax no demostró mayor hallazgo (Fig. 2) y la ecovisión revela $\mathrm{FEVI} 20 \%$ con derrame pericárdico y una masa hiperecogénica compatible con coágulo adherido a la pared (Fig. 3). El diagnóstico planteado fue estado de bajo gasto cardiaco por infarto de miocardio no $Q$ evolutivo con rotura miocárdica.

Ingresa a ventilación mecánica, el AGA de control mostró acidosis mixta con lactato en $6.3 \mathrm{mmol} / \mathrm{L}$, se aumentó el volumen minuto y se mantuvo niveles de presión positiva al final de la espiración (PEEP) menores a $5 \mathrm{cmH} 2 \mathrm{O}$. La paciente cursó con episodios de bradicardia sinusal con pausas y latido de escape nodal sobre un ritmo sinusal y frecuencia cardiaca en 62 latidos por minuto. En la ecografía transtorácica de control se halló derrame pericárdico de $250 \mathrm{ml}$ con colapso de cavidades derechas, no se aprecia shunts, no alteraciones valvulares significativas. Se procede a realizar pericardiocentesis obteniéndose 150 cc de líquido serohemático. Cuarenta minutos después la paciente entra en paro cardiorespiratorio, asistolia en dos oportunidades sin éxito en las maniobras de reanimación.

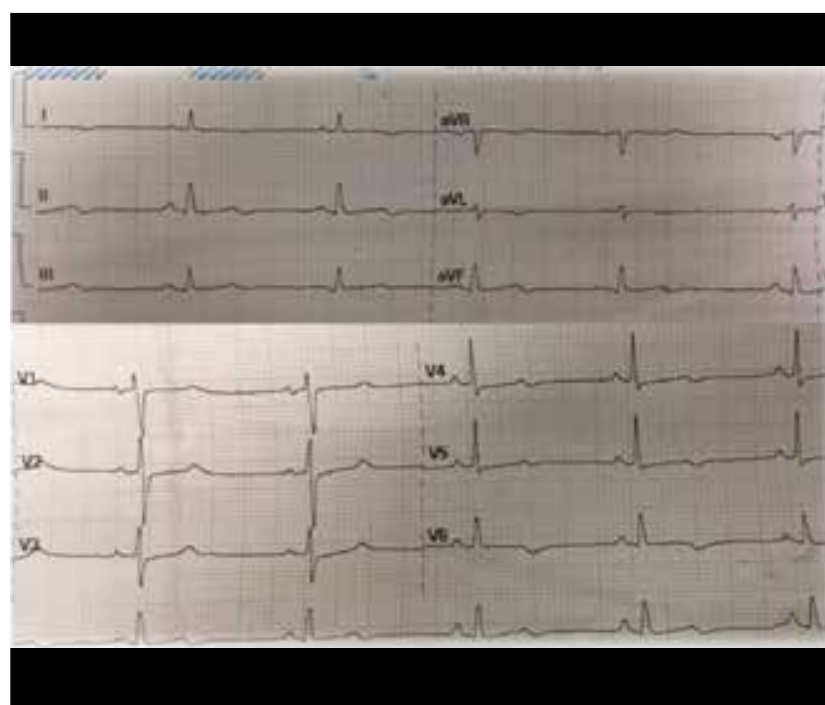

Figura 1. Electrocardiograma. Ritmo sinusal, FC: 78x', eje $40^{\circ}$, no elevación del ST, no presencia de onda $\mathrm{Q}$.

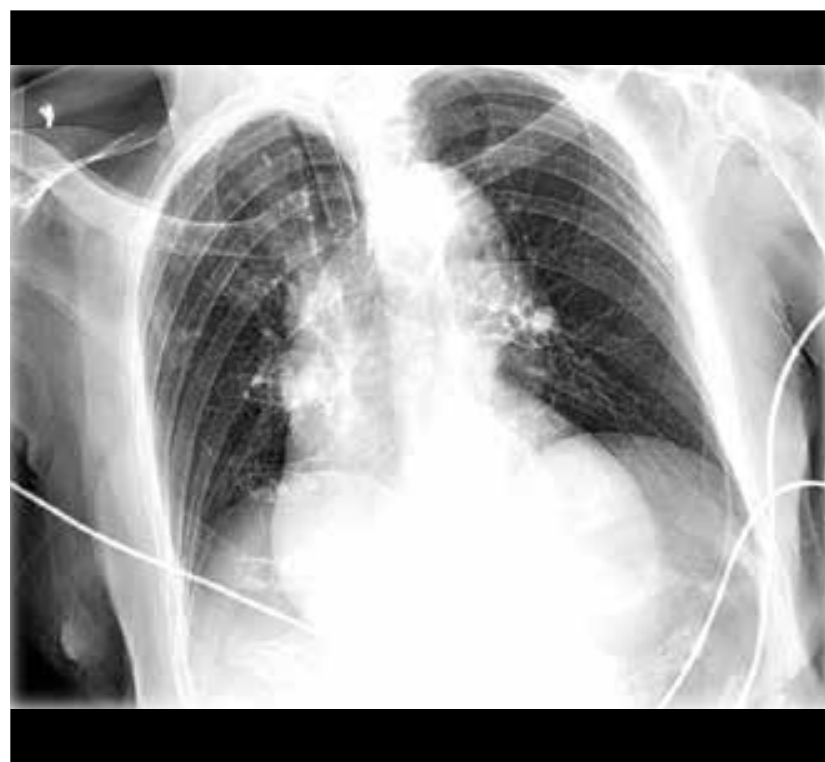

Figura 2. Radiografía de tórax (Rx-T): La Rx-T frontal (17.XII.17) muestra parénquima pulmonar sin nódulos ni masas, no se evidencia engrosamiento intersticial ni bronquiectasias ni panalización. No efusión pleural.

\section{DISCUSIÓN}

La rotura miocárdica es una complicación mecánica fatal de un infarto agudo de miocardio (IMA) que es directamente responsable de la mortalidad en el $8 \%$ de los pacientes infartados. La ruptura de la pared libre de ventrículo izquierdo, ocurre en aproximadamente el $2 \%$ de los casos, pero hoy en día, en la era de la intervención coronaria percutánea (ICP), es menos frecuente ${ }^{3}$. El $40 \%$ de los casos se produce en las primeras 24 horas del evento isquémico agudo y el $85 \%$ dentro de la primera semana ${ }^{1,2,4}$. La ruptura no suele ocurrir después de 10 días, cuando la curación ha tenido lugar'. 

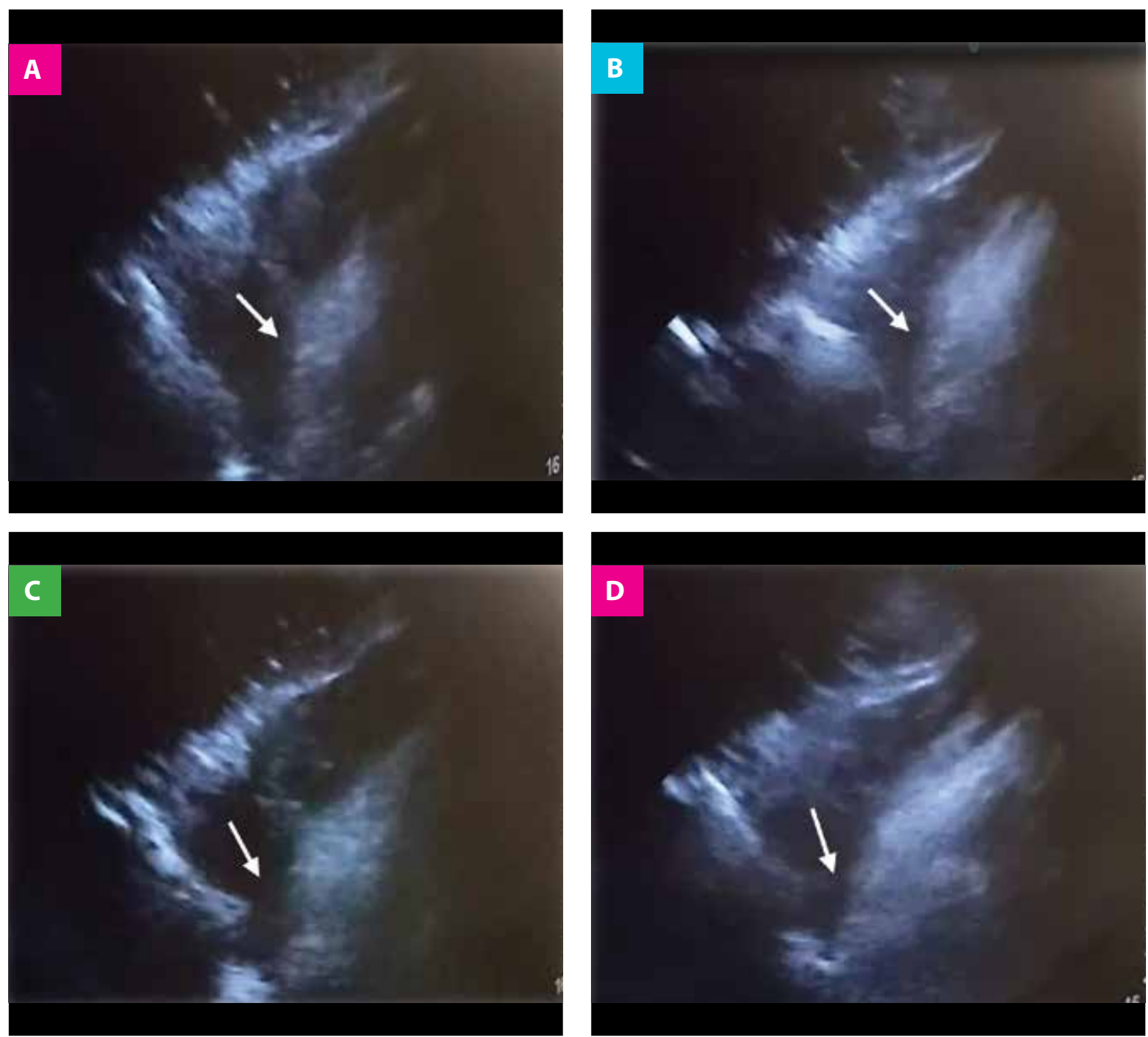

Figura 3. Ecocardiograma (17.XII.17). Imagen A, B, C Y D: Derrame pericárdico de aproximadamente 250cc (prepericardiocentesis) con compromiso hemodinámico, ventrículo izquierdo dilatado con colapso de Ventrículo derecho (flecha blanca). No se aprecia shunts. No alteraciones valvulares significativas.

No se ha reportado hallazgo físico específico para rotura cardiaca debiéndose pensar en ello cuando existe un rápido deterioro de la condición clínica como en el presente caso. La ecocardiografía de cabecera es el método de diagnóstico más rápido ${ }^{5}$.

Un alto índice de sospecha y diagnóstico temprano es de vital importancia para la sobrevivencia de estos pacientes. Se consideran factores predisponentes; como presentaba la paciente; edad avanzada, sexo femenino, hipertensión arterial sin ataques anginosos previos y dolor en el pecho persistente o recurrente. Se considera también como factores de riesgo un primer infarto transmural anterior o lateral sin síntomas de insuficiencia cardíaca manifiesta y elevación persistente del segmento $\mathrm{ST}^{2,3}$. La paciente ingresó con cuadro clínico compatible con síndrome coronario agudo y ya desde el ingreso estuvo en mal estado general, hipotensa, confusa y con signos de shock por lo que también se debe plantear en estos casos la posibilidad de rotura cardíaca. En ese entorno, la rotura de la pared libre es propensa en los pacientes con un infarto de miocardio transmural que involucra la arteria descendente anterior y circunfleja. La administración de agentes trombolíticos puede promover la rotura cardíaca, presumiblemente debido a una tendencia hemorrágica ${ }^{2}$. El tiempo de la rotura libre de pared ha disminuido de 3 a 5 días en la era pretrombolítica a 12 a 24 horas en la era trombolítica. Por el contrario, la ICP reduce la incidencia de la rotura miocárdica y mejora la mortalidad y morbilidad debido al IMA ${ }^{6}$.

Existen tres formas clínicas de rotura de la pared libre ventricular. La más frecuente es la rotura aguda 
(70\% de los casos) seguida de hemorragia masiva intrapericárdica y taponamiento de evolución fatal en pocos minutos sin tiempo suficiente para intervenir al enfermo ${ }^{4,6}$. En segundo lugar, la rotura subaguda que se caracteriza porque el taponamiento se instaura con menos brusquedad (debido a un desgarro incompleto) y el paciente puede sobrevivir varias horas. Se calcula que más de la mitad de los pacientes con rotura subaguda fallecen durante las primeras 6 horas tal como ocurrió en el presente caso. Se han descrito casos excepcionales de rotura subaguda con supervivencia a largo plazo sin tratamiento quirúrgico. En tercer lugar, la rotura puede pasar desapercibida en la fase aguda del infarto, probablemente al ser contenida por adherencias pericárdicas preexistentes, dando lugar a la formación de un falso aneurisma crónico ${ }^{2,4,6}$. El cuadro clínico se caracteriza por el colapso cardiovascular con disociación electromecánica con un desenlace mortal en pocos minutos ${ }^{2,3}$. Sin embargo, en un $25 \%$ de los casos la presentación es subaguda (debido a trombos o adhesiones que la sellan) lo que facilita la intervención ${ }^{3}$. Los pacientes pueden o no tener síntomas premonitorios, pero si están presentes, presentan dolor torácico persistente, agitación y emesis recurrente ${ }^{6}$. La rotura subaguda de la pared libre de ventrículo izquierdo puede simular el del reinfarto por la recurrencia del dolor y nuevas elevaciones del segmento ST, aunque más frecuentemente se presenta como un deterioro hemodinámico súbito con taponamiento cardiaco, hipotensión persistente y estado de bajo gasto ${ }^{2,7,8}$. Esta forma tiene un pronóstico muy grave, con necesidad de intervención quirúrgica urgente, y se asocia a alta mortalidad, hasta el $80 \%$ si hay rotura de pared libre 2 .

Los cambios en el electrocardiograma en pacientes con rotura cardíaca varían de normal a elevación del ST anteroseptal con desviación del eje a la izquierda, elevación del ST anterior, cambios típicos de IMA inferior o de IMA posterior, seudonormalización de onda $\mathrm{T}$, elevación del segmento $\mathrm{ST}$ en $\mathrm{AVL}$, nuevas ondas $\mathrm{Q}$ en al menos dos derivaciones y cambios no específicos en el ST-T. Si bien es cierto no se presentaron cambios importantes en el electrocardiograma (EKG), la paciente tuvo elevación de troponina importante. La elevación persistente de ST o una desviación positiva de la onda $T$ que persiste más de 72 horas, no es sensible ni específica de ruptura subaguda 6 . El estado final de la rotura se corresponde con bradicardia y disociación electromecánica, método diagnóstico siempre importante pero no determinante en caso de rotura cardiaca ${ }^{1}$.
La ecocardiografía es una técnica sensible para diagnosticar la rotura miocárdica, aunque la presencia del derrame por sí solo no es suficiente para diagnosticarla, ya que puede estar presente en el $28 \%$ de los pacientes tras un síndrome coronario agudo y rotura subaguda, ${ }^{5,6}$. La ecografía para diagnosticar la ruptura ventricular tiene70\% o más de sensibilidad y $90 \%$ de especificidad; los hallazgos incluyen fluido pericárdico ecodenso de más de 5 $\mathrm{mm}$ de espesor y hallazgos de colapso diastólico ventricular derecho, vena cava inferior dilatada $y$ marcada variación respiratoria en el flujo de la entrada de las válvulas tricúspide y mitral ${ }^{4,7,8}$. La presencia de masas ecogénicas aumenta tanto la sensibilidad (97\%) como la especificidad (93\%). Asimismo, el derrame pericárdico moderado a grave $(>10 \mathrm{~mm})$ en el paciente con IMA ST elevado se asocia con el $43 \%$ de la mortalidad a los 30 días $^{6}$. Por lo tanto; el derrame no aumenta la morbilidad y la mortalidad en el infarto de miocardio, pero sirve como otro indicador de un infarto de gran área. Derrames mayores o derrames densos ecocardiográficos que representan hemorragia siempre deben hacer que los médicos consideren una ruptura de la pared libre?

El uso de la pericardiocentesis de emergencia es controversial. Pensado como contribuyente al estado de shock de la paciente se practicó el procedimiento. Su indicación en pacientes que cursan con taponamiento cardíaco constituye una medida de rescate para pacientes que esperan manejo quirúrgico casi inmediato; sin embargo, puede provocar un incremento de la tensión del miocardio dañado y potencial extensión del área de rotura cardiaca ${ }^{1,7,8}$. El tratamiento inicial está dirigido a estabilizar el paciente e incluye oxigenación/ventilación fluidos intravenosos con pequeños volúmenes e inotrópicos ${ }^{6}$.

La ecocardiografía es una valiosa herramienta de diagnóstico no invasiva que puede proporcionar información sobre la función cardíaca y las anomalías valvulares, y explicaciones alternativas para las causas del dolor torácico y la dificultad para respirar. Se reconoce ampliamente que la ecocardiografía de emergencia es la técnica de imagen más versátil y rentable para evaluar a los pacientes con enfermedades cardiovasculares inestables ${ }^{9,10}$. Es la prueba más comúnmente disponible y puede ser realizada por el médico de urgencias en la sala de reanimación.

A pesar del alto riesgo quirúrgico, la reparación quirúrgica sigue siendo el estándar de oro para controlar esta rara afección ${ }^{11}$. Las actuales guías del Colegio americano de cardiología y la Asociación 
americana del corazón ACCF/AHA (American College of Cardiology Foundation and American Heart Association) recomiendan la reparación quirúrgica de emergencia independientemente de la estabilidad hemodinámica en el momento del diagnóstico. A pesar del acuerdo general entre los expertos sobre la necesidad de reparación quirúrgica, el tiempo de la reparación y el manejo terapéutico perioperativo son controversiales ${ }^{7,8}$. En el caso presentado la paciente no llegó a ingresar a sala de operaciones habida cuenta del tiempo que tomó el desarrollo de los acontecimientos. Existen sin embargo algunos puntos controversiales, en todos los casos ha de ser implementada la cirugía?, la revascularización puede ser concomitante o no con la cirugía? o, se puede retardar la cirugía?.

\section{CONCLUSIÓN}

Este caso resalta de manera excepcional el papel de las técnicas diagnósticas no invasivas, en especial el uso de la ecocardiografía en todo paciente con síndrome coronario agudo ${ }^{1,9}$ y asimismo el tener en cuenta esta patología para un diagnóstico y tratamiento oportuno.
Agradecimiento: Agradecemos al Instituto de Evaluación de Tecnologías en Salud e Investigación (IETSI) de EsSalud por el soporte para el desarrollo de este manuscrito a través de su Programa de Mentoring.

Contribuciones de autoría: JC, RV y FR han participado en la concepción y diseño del artículo y aprobación de la versión final del manuscrito. JC elaboró el primer borrador del artículo. JC, RV y FR han realizado la revisión crítica del contenido.

Financiamiento: Autofinanciado.

Conflicto de interés: Al momento de la concepción y de la publicación del artículo los autores laboraron en EsSalud.

Recibido: 6 de noviembre 2018

Aprobado: 27 de diciembre 2018

\section{Correspondencia: Jessica M. Calcino Cuela}

Dirección: Servicio de Emergencia Hospital Rebagliati, Seguro Social-EsSalud. Domingo Cueto N¹20, Jesús María. Lima, Perú

Celular: +51999242524

Correo:jessica_milcc@hotmail.com

\section{REFERENCIAS BIBLIOGRÁFICAS}

1. Wilansky S, Moreno CA, Lester SJ. Complications of myocardial infarction. Crit Care Med. agosto de 2007; 35(8 Suppl):S348-354.

2. Facenda-Lorenzo M, Poncela-Mireles FJ, Álvarez-Acosta L, Gómez-Ferrera $\mathrm{N}$, Trugeda-Padilla A, Llorens-León R. Rotura cardiaca contenida como hallazgo ecocardiográfico casual tras infarto inferolateral evolucionado. Cir Cardiovasc. 1 de enero de 2014; 21(1):57-9.

3. Abdelnaby M, Al-Maghraby A, Saleh Y, El-Amin A, Haleem MMA, Hammad B. Post-Myocardial Infarction Left Ventricular Free Wall Rupture: A Review. Ann Med Health Sci Res. 2017; 7:368-372.

4. Joaquín J, Azpitarte J, Bardají A, Cabadés A, Fernández A, Palencia M, Permanyer $C$ y Rodríguez E. Guías de práctica clínica de la Sociedad Española de Cardiología en cirugía coronaria. Rev Esp Cardiol 2000; 53: 241-266.

5. Gulalp, B., Karagun, O., Tekin, A., Benli, S. Cardiac Rupture. The Journal of emergency edicine, 44(1), 58-60. 2013

6. Anurag B, Ankur S, Parul R, Nissi S, Arjinder S. Acute Complications of Myocardial Infarction in the Current Era: Diagnosis and Management. J InvestigMed 2015; 63: 844-855
7. Quitian J, Ariza J, Rugeles Ty Bermúdez L. Complicaciones mecánicas del infarto agudo de miocardio: aunque infrecuentes, potencialmente letales. Rev Colomb Cardiol. 2017; 24(5):505-509

8. Kutty RS, Jones N, Moorjani N. Mechanical Complications of Acute Myocardial Infarction. Cardiol Clin. 1 de noviembre de 2013; 31(4):519-31.

9. Lancellotti P, Price S, Edvardsen T, Cosyns B, Neskovic AN, Dulgheru $\mathrm{R}$, et al. The use of echocardiography in acute cardiovascular care: recommendations of the European Association of Cardiovascular Imaging and the Acute Cardiovascular Care Association. Eur Heart J Acute Cardiovasc Care. Febrero de 2015; 4(1):3-5

10. Kim T-S, Youn H-J. Role of Echocardiography in Atrial Fibrillation. J Cardiovasc Ultrasound. Junio de 2011; 19(2):51-61.

11.Durko AP,BuddeRPJ,GeleijnseML, Kappetein AP.Recognition, assessment and management of the mechanical complications of acute myocardial infarction. Heart. 16 de noviembre de 2017; heartjnl-2017-311473. 